\title{
Tumor microenvironment-aware, single- transcriptome prediction of microsatellite instability in colorectal cancer using meta-analysis
}

\author{
Mi-Kyoung Seo \\ Yonsei University College of Medicine \\ Hyundeok Kang \\ Yonsei University College of Medicine \\ Sangwoo Kim ( $\square$ swkim@yuhs.ac) \\ Yonsei Univ. College of Medicine
}

\section{Research Article}

Keywords: Microsatellite instability, Colorectal cancer, Recursive feature elimination-random forest, Tumor microenvironment, Machine learning

Posted Date: November 15th, 2021

DOI: https://doi.org/10.21203/rs.3.rs-1019124/v1

License: (9) This work is licensed under a Creative Commons Attribution 4.0 International License.

Read Full License

Version of Record: A version of this preprint was published at Scientific Reports on April 15th, 2022. See the published version at https://doi.org/10.1038/s41598-022-10182-3. 
1 Tumor microenvironment-aware, single-transcriptome prediction of

2 microsatellite instability in colorectal cancer using meta-analysis

3

4 Mi-Kyoung Seo ${ }^{1,2}$, Hyundeok Kang ${ }^{1}$, Sangwoo Kim ${ }^{1 *}$

5

$6{ }^{1}$ Department of Biomedical Systems Informatics and Brain Korea 21 PLUS Project for

7 Medical Science, Yonsei University College of Medicine, Seoul 03722, South Korea

$8{ }^{2}$ Department of Nuclear Medicine, Seoul National University Hospital, Seoul 03082, South

9 Korea

10

$11 *$ Correspondence

12 Sangwoo Kim

13 Postal address: \#613, Yonsei Univ. College of Medicine, 50-1 Yonsei-ro, Seodaemun-gu, Seoul

14 03722, South Korea

15 E-mail: swkim@yuhs.ac

16 Telephone: +82-2-2228-2589

17

18

19 
21 Detecting microsatellite instability (MSI) in colorectal cancers (CRCs) is essential since it is therapeutic strategy determinant feature, including immunotherapy and chemotherapy. Yet, no attempt has been made to exploit transcriptomic profile and tumor microenvironment (TME) of it to unveil MSI status in CRC. Hence, we developed a novel TME-aware, singletranscriptome predictor of MSI for CRC, called MAP (Microsatellite instability Absolute single sample Predictor). MAP was developed utilizing recursive feature elimination-random forest with 466 CRC samples from The Cancer Genome Atlas, and its performance was validated in independent cohorts, including 1118 samples. MAP showed robustness and predictive power in predicting MSI status in CRC. Additional advantages for MAP were demonstrated through comparative analysis with existing MSI classifier and other cancer types. Our novel approach will provide access to untouched vast amounts of publicly available transcriptomic data and widen the door for MSI CRC research and be useful for gaining insights to help with translational medicine.

Keywords: Microsatellite instability; Colorectal cancer; Recursive feature elimination-random forest; Tumor microenvironment, Machine learning 
Microsatellite instability (MSI) is characterized by genetic hypermutability due to impaired DNA mismatch repair (MMR) system ${ }^{1}$. MSI is observed in many solid tumors, including gastric, and endometrium cancers, as well as in colorectal cancer (CRC, approximately $15 \%)^{2,3}$. Exhibiting prognostic and predictive features of a high tumor mutational burden, a high neoantigen load, and an immune-active tumor microenvironment (TME) characterized by high levels of tumor-infiltrating lymphocytes and overexpression of immune checkpoint molecules, cancers with MSI are known to be great candidates for immune checkpoint inhibitors (ICIs) treatment, such as pembrolizumab and atezolizumab (anti-PD-1 and anti-PD-L1 monoclonal antibody, respectively $)^{4,5}$. In addition, MSI primary tumors face a better prognosis than patients with microsatellite stability (MSS) tumors ${ }^{2}$. MSI status is meaningful as a predictive indicator for cancer treatment and as a prognostic determinant, identifying a patient's MSI status is essential in clinical setting and research areas.

With recent escalation of its importance in $\mathrm{CRC}$, it has been explored from publicly obtained samples, such as The Cancer Genome Atlas (TCGA) and Gene Expression Omnibus (GEO) database, resulting in numerous studies which broaden our understanding in MSI and expand therapeutic options for MSI CRC patients ${ }^{6}$. However, prior to utilization, MSI status information must be provided beforehand by quantifying the extent of genomic events in microsatellite loci, at genomic level, using the Bethesda Panel, a PCR-based five marker panel, or by examining the loss of mismatch repair proteins using immunohistochemistry (IHC) at the protein level ${ }^{1}$. Additionally, with recent advances in next-generation sequencing (NGS) technology, MSI predictors, such as MANTIS $^{7,8}$, MSIsensor $^{9}$, and MOSAIC ${ }^{10}$, have been developed to extract MSI status from whole exome and whole genome sequencing data. However, assigning the MSI status from expression data had not been possible until a k-Nearest Neighbors ( $\mathrm{k}-\mathrm{NN}, \mathrm{k}=5$ ) classifier called preMSIm using 15 gene-set for pan-cancer was 
recently proposed ${ }^{11}$ and several other attempts which had been made, although the software has not been made readily available ${ }^{12,13}$.

The preMSIm has constructed as a pan-cancer MSI predictor by utilizing three MSI dominant carcinomas as training data ${ }^{11}$, but it did not reflect the distinct expression profiles of its cancer of origin. Furthermore, individual MSI tumors have unique tumor microenvironment (TME) and molecular pathway characteristics. For example, immune inflamed MSI microenvironment could be characterized by higher infiltration of anti-tumorigenic immune cells, such as adaptive and natural killer cells) than immune desert MSS tumors, and, in CRC, when mutations or activation of MYC and RAS pathways occur, chemokine CCL9 is expressed and an immunosuppressive environment is established, which prevents enrichment of cytotoxic NK cells and $\mathrm{T}$ cells around the tumor ${ }^{14}$. Therefore, transcriptome based MSI predictor which integrates both TME and molecular pathway characteristics in CRC is needed.

Here, in this study, we have developed an enhanced single-sample MSI classifier called MAP (Microsatellite instability Absolute single sample Predictor) that integrates transcriptomic characteristics of TME and molecular pathways to predict MSI in CRC. Our TME and molecular pathways aware predictor will open a way to utilize CRC expression data to elucidate MSI CRC. Hence, massive amounts of publicly available expression data without MSI status will be utilized to drive valuable MSI CRC research through our novel approach, and, furthermore, to give patient benefit in clinical setting.

\section{Results}

\section{Overview of MAP development}

As an MSI single sample predictor (SSP), the MAP model was developed with the following 
four components (Fig. S1): 1) identification of the MAP signature (MAPgene model); 2) modeling based on pairwise gene expression of the MAP signature genes (MAPpairs model); 3) modeling based on ssGSEA scores of cancer-, molecular-, TME-, and immune-related signatures (MAPsig model); and 4) post-refinement of the final model and prediction of MSI status. To develop an SSP of MSI status without relying on a relative approach (e.g., comparing a patient's data with other samples) and with limited platform bias, we constructed a recursive feature elimination-random forest (RFE-RF) model (MAPpairs model) with pairwise gene comparisons, leveraging an informative gene-set (MAP signature from the MAPgene model), rather than gene expression profiles, on a training dataset. In brief, RFE trains the model, ranks the features, and selects features through the process of repeatedly removing lower-ranked features ${ }^{15}$. The method of building a model by selecting features with the RFE method based on the RF algorithm is called RFE-RF ${ }^{15}$. We built another RFE-RF model (MAPsig model) based on ssGSEA scores for 101 signatures to reflect the degree of activity of cancer-, immuneand TME-related signatures of the samples. To select the best RFE-RF model from the parts mentioned above, we evaluated the area under the receiver operating characteristic curve (AUC) and confirmed the model performance in validation datasets (Table S1). Finally, at the postrefinement stage, an integrated MAPpairs and MAPsig model was used to precisely predict MSI status. We named this final model MAP and evaluated its accuracy, kappa, sensitivity, specificity, F1, and balanced accuracy in the validation datasets (Table S2).

\section{MAP signature}

To minimize the size of the informative gene-set utilized in the MAPgene model, we, first, identified differentially expressed genes (DEGs) between MSI and MSS samples using the Wilcoxon rank-sum test. We assessed 718 DEGs with criteria of $P<0.001$ and $\mid \log _{2}$ fold change $\mid>1$, and selected a gene-set of 31 genes by performing RFE-RF modeling with an AUC 
of $99.2 \%$. We called this gene-set the MAP signature (11 up- and 20 down-regulated DEGs, Fig. 1a and Table S3). Among genes comprising the MAP signature, the $M L H 1$ gene, which is commonly downregulated and/or hypermethylated in sporadic MSI samples, ranked as the top feature gene, based on both accuracy (the importance of the features that improves classification accuracy) and Gini index values (the importance of the features that reduces the impurity of classification) (Fig. $1 \mathrm{~b}$ and Table S3). We also noted that $L Y 6 G 6 D^{16}$ and $E P M 2 A I P 1$ genes $^{17}$, which share a promoter with $M L H 1$, were included in the gene-set. Additionally, we found that a known predictive marker for chemotherapy, thymidylate synthase $(T Y M S)^{18}$, was included in the gene-set, and its expression was higher in the MSI samples than the MSS samples (Fig. 1a and 1b). Other genes belonging to the following pathways were also included in the MAP signature: the WNT signaling pathway (RNF43, TCF7, and NKD1), Hippo signaling (TCF7, NKD1, and TGFBR2), and MAPK signaling (DUSP4 and TGFBR2). Three well-known frameshift mutated genes (DDX27, TGFBR2, and RNF43) in microsatellite loci in MSI CRC were also included. In terms of MMR, 718 DEGs were initially used when constructing the MAPgene model, although three MMR genes (MSH2, MSH6, and PMS2) were not included because their statistical significance or fold change did not meet the inclusion criteria (Fig. S2).

To assess the representativeness of the MAP signature (31-genes) in reflection of MSI status, the expression patterns of the genes in the gene-set were investigated in a validation dataset, and expression patterns similar to those observed in the discovery dataset were noted. To further investigate whether the MAP signature could serve as a surrogate marker of MSI status and to evaluate its generalizability, we obtained and compared ssGSEA scores for the MAP signature in MSI and MSS tumors, as well as in MSI tumors of each of the four consensus molecular subtypes (CMSs). The general comparison between MSI and MSS samples revealed significant differences in MAP signature scores $\left(P=7.6 \times 10^{-36}\right)$, but not among the CMSs (Fig. 
1c). This suggests that the composite genes of the MAP signature can capture MSI's behaviorrelated features and discriminate between MSS and MSI status independent of CMS context.

\section{MAP model}

Although the MAPgene model built based on gene expression showed high performance, to develop a true SSP with unnormalized data that does not rely on a relative comparison among multiple samples, we employed a pairwise gene comparison approach for model building: for example, if the expression of gene A was greater than that of gene B, the sample would be assigned MSI status. An RF model with 1000 trees of such rules was constructed utilizing the RFE-RF algorithm with five-fold cross-validation repeated 100 times. Finally, the MAPpairs model, comprised of 187 rules from $465\left({ }_{31} \mathrm{C}_{2}\right)$ rules at a starting point, was selected (AUC of 99.7\%). To assess its performance and reproducibility, we applied the model to internal and external RNA-seq validation datasets and obtained accuracies of $99.1 \%$ and $95.4 \%$, respectively, indicating it to be robust and highly accurate. In the MAPpairs model, MLH1related features (MLH1/HPSE, MLH1/FECH, and MLH1/GNLY) were the highest ranked features (Fig. 2a), and the expression levels of these features separated the two groups well (Fig. 2b). To investigate the features of MAPpairs further, we calculated the number of MSI and MSS samples that satisfied each rule in MAPpairs (Fig. S3). Most rules were able to classify MSI and MSS samples, and as such, they were considered to be reflective of the overall characteristics of MSI, although not all samples may show similar profiles: For example, MSI samples are known to have a loss of $M L H 1$ and an immunity-activated characteristic ${ }^{2}$, as well as high expression of thymidylate synthase (TYMS) (chemotherapy response-associated gene) ${ }^{18}$. Features of the MAPpairs model, $M L H 1 / G N L Y$ and TYMS/MLH1, respectively, described these MSI characteristics well (Fig. 2b), but not in all tumors. This may suggest that the MAPpairs model, a random forest classifier, captures and reflects the more complexity of MSI CRC, not 
just a simple single rule.

In order to complement the MAPpairs model with the characteristics of immune and TME profiles, as well as the transcriptomic profile and tumor's characteristics of MSI, we built the MAPsig model based on molecular, cancer, immune, TME, and MAP signature scores inferred by single-sample gene set enrichment analysis (ssGSEA). The top signatures used in the final MAPsig model (44 signatures) included the MAP signature, antitumorigenic immune lymphocytes (effector memory CD8 T cell, Teff (CD8 T effector), Th2 cells, activated CD4 T cell), complement, INF- $\gamma$ signatures, Wnt- $\beta /$ catenin signaling, glycolysis, and cell cycle signaling (Fig. 2c). To find out the degree of activation of 44 signatures, we investigated the heatmap based on the inferred ssGSEA score. Compared to MSS, antitumorigenic immune lymphocytes, complement, glycolysis, cell cycle, and INF- $\gamma$ related signatures were upregulated in MSI, whereas MAP signature, Notch, angiogenesis, epithelial signature, and Wnt$\beta /$ catenin signaling were down-regulated (Fig. S4). The final MAP model was established after integrating the MAPpairs and the MAPsig models, and post-refinement processing was done by utilizing probability. Next, we applied the final MAP model to validation datasets to evaluate any potential overfitting and its applicability across multiple platforms. A total of 1118 samples (240 MSI and 878 MSS tumors) were tested, and MAP exhibited an average accuracy of $96.1 \%$ (95\% confidence interval (CI) $94.3-98.9$ ), a sensitivity of $93.1 \%$, a specificity of $97.5 \%$, and an F1 score of $92.0 \%$ (Fig. $2 \mathrm{~d}$ and 2e), indicating outstanding performance and feasibility as an MSI predictor.

\section{MSI signatures in other cancer types}

Using TCGA-STAD and TCGA-UCEC RNA-seq datasets, we evaluated whether MAP, which was developed for CRC, could be applied to other cancers. In the stomach adenocarcinoma (STAD) and uterine corpus endometrial carcinoma (UCEC) data, accuracies of $80.2 \%$ and $75.4 \%$ 
were observed, respectively. To investigate why the MSI classifier of CRC is not suitable for other cancers, the same method used to construct the MSI signature (MAP signature) in CRC was applied to examine MSI signatures in gastric cancer and uterine cancer, and then the differences in expression patterns were investigated. Uterine cancer showed an accuracy of 90.9\%, with only nine genes (CXCL13, EPM2AIP1, H2AFJ, HOXA9, MLH1, RNLS, SDR42E1, TNFSF9, and ZNF300), whereas gastric cancer reached an accuracy of $83.4 \%$ using 78 genes (Table S4). We further probed how cancer-specific MSI signatures are expressed in each cancer and observed that individual MSI signatures tend to correspond to DEGs not statistically significant in other cancers (Fig. 3a and 3b). MLH1 and EPM2AIP1 were differentially expressed in all three cancers, $R P L 22 L 1$ was included in the MAP signature of CRC and STAD, and $H 2 A F J$ was observed in both CRC and UCEC. In addition, comparing the MAP signature and MSI signature from the recently developed preMSIm, five genes (MLH1, RPL22L1, EPM2AIP1, DDX27, and SHROOM4) were observed in both signatures in CRC (Fig. 3c). It is also worth mentioning that all of the genes used in preMSIm are down-regulated in MSI, except RPL22L1, whereas the MAP signature additionally includes both up- and down-regulated genes in CRC. In addition, the expression pattern of the signature of preMSIm did not appear to suitably reflect genes important in gastric cancer, such as DDX27, SMAP1, and ZSWIM3, and in uterine cancer, such as DDX27, SHROMM4, SMAP1, and ZSWIM3, thereby making it unable to efficiently differentiate MSI and MSS tumors $\left(\mid \log _{2}\right.$ fold change $\left.\mid<0.5\right)$ in these cancer types (Fig. 3d and 3e).

\section{Discussion}

Not all MSI status information is available in publicly available colorectal cancer expression data, such as NCBI Gene Expression Omnibus (NCBI GEO), thus such data cannot be utilized in MSI CRC research. For example, it hampers studies determining why most MSS samples 
214 belong to the immune desert type or the mechanism by which immune evasion occurred in a 215 subset of MSI tumors by utilizing molecular or immunological characterization of MSI and MSS. Although at the research level, if these studies are conducted, this may give clues to convert the immune-inactivated tumors into immune-activated types or to discover drugs targeting abnormally activated oncogenic pathways or suppressed TMEs which can be combined with ICIs. Additionally, since MSI samples are rare, with the difficulty of producing expression data due to RNA degradation, meta-analysis using multiple cohorts is required, but the use is hindered due to the absence of MSI status information in them. Furthermore, MSI research analysis can be performed after cross-validation of the MSI status of RNA-seq data of the tumor identified as the MSI sample at the DNA level. Here, we present MAP, a tumor microenvironment-aware, single-transcriptome predictor of MSI in CRC, with robust accuracy validated using large samples from multiple cohorts of primary tumors. $(N=1118)$. We expect that the MAP will open the door to make such datasets of use in future MSI studies. MAP has the advantage of not requiring a matched normal sample as a control and sufficiently predicts MSI status with a single-sample transcriptome profile.

Attempts to create an absolute predictor for subtype classification of cancer and stratification of patients by applying relationships or ratios between two genes, not the expression value of the gene itself, are ongoing ${ }^{19,20}$. MAP is an absolute classifier, not relative, and was developed to reflect tumor molecular characteristics, immune-related signatures, and tumor-infiltrating immune cells in TME of CRC. Also, since MAP is an RF classifier, one feature does not represent all MSI in common, but the MSI status is determined through the complex reflection of various features. Therefore, it may be difficult to interpret clinical and biological significance of features, and it might be considered to be included technical as well as biological rules to improve the accuracy of classification. 
of MSI in the internal validation TCGA dataset. Only one sample (TCGA-DC-6154) with MSI status was incorrectly predicted as being MSS by the MAP model, and it was also marked as MSS with the MOSAIC program, a tool which predicts MSI status at genomic level ${ }^{10}$. We speculated such discrepancy may stem from the different tissue sampling locations (MSI typing vs. DNA and RNA sequencing) or MSI intratumor heterogeneity, rather than MAP misinforming. We also encountered misclassification of a 11CO070 (MSS) hypermutated sample from an external RNA-seq validation dataset and five MSS samples from the GSE39582 dataset as MSI by MAP. Using the clinical information available, we further investigated the five MSS samples from the GSE39582 dataset and they all carried BRAF mutation and high $\mathrm{CpG}$ island methylator phenotype (CIMP). In sporadic MSI CRC, the accompanying characteristics of BRAF mutation and high CIMP are known to be strongly correlated with $\mathrm{MSI}^{21}$, but it was not possible to determine misassignment or tumor heterogeneity characteristics in detail due to the absence of lynch syndrome status or mutation information of other MMR genes of the samples. Additionally, in research on CMS reported by the Colorectal Cancer Subtyping Consortium, the distribution of CMS2 (known as immunedesert type) samples with MSI status was exceedingly rare (10 of 270, 2.7\% $)^{2}$, whereas eight out of the 10 CMS2-MSI samples belonged to one cohort (GSE13294 dataset). This particular cohort carried a slightly dissimilar CMS2-MSI population distribution from the other datasets, and out of these eight samples, five were classified as MSS by MAP. MAP showed accuracies of $98.6 \%$ (95\% CI 97.6-99.6) in RNA-seq and 95.1\% (95\% CI 91.698.7) in microarray data, all primary tumor and MSI detected based on PCR panel, showing a slight difference depending on the platform. Although MAP is an absolute SSP with a specificity of approximately $97 \%$ and a high accuracy of $96.1 \%$, it may be due to the inherent characteristics derived from development based on RNA-seq, or a rare MSI subgroup (eg. immune-desert CMS2-MSI) that exists in a specific cohort (GSE13294). Due to the paucity of 
clinical information, we were unable to thoroughly characterize the samples that were not accurately predicted.

The recently developed preMSIm, a pan-cancer MSI predictor, is a k-NN classifier using 15 genes identified by using only three frequent cancer types (COAD, STAD, and UCEC) as training data. However, due to the limitations mentioned by the author of preMSIm ${ }^{11}$ and based on our findings, these 15 genes are not enough to predict MSI in pan-cancer. This is because tumor biology and tumor microenvironment are distinct for individual cancer origins, suggesting diverse tumor-intrinsic gene expression patterns. In this context, MAP is superior when predicting the MSI status in CRC as it was designed to reflect both the molecular characteristics of CRC and the complexity of its surroundings.

As the MAP model includes the $M L H 1$ gene, sporadic CRC, characterized by $M L H 1$ promoter hypermethylation or MLHI loss, can be classified well, whereas Lynch syndrome, a familiar syndrome, due to germline mutations of MMR or EPCAM gene ${ }^{1}$, may not be reflected. In addition, due to the lack of IHC and clinical information (e.g., KRAS, BRAF mutations, and for clinical application, MAP will find use in MSI-related research seeking to employ the large amounts of publicly available CRC expression data and will be useful for gaining insights to help with translational medicine. 


\section{Methods}

292

293

294

295

296

297

298

299

300

301

302

303

304

305

306

307

308

309

310

311

312

313

\section{Dataset acquisition}

This meta-analysis was performed in accordance with the PRISMA guidelines (Fig. S5). For the discovery cohort, 581 RNA-seq data (rsem.norm.expression) from TCGA-COADREAD were downloaded from the TCGA data portal (https://portal.gdc.cancer.gov/). Matching data on MSI status (82 MSI and 499 MSS) was downloaded from The Cancer Imaging Archive (TCIA) (https://tcia.at/). For the validation cohort, 106 RNA-seq data from 24 MSI and 82 MSS samples (rsem.norm.expression) from an independent study ${ }^{22}$ were downloaded. MSIlow tumors were grouped with MSS tumors as in previous studies ${ }^{23,24}$. The gene expression values of RNA-seq were log-transformed (with base 2) for analysis. Five independent microarray-based cohorts were used as an additional validation dataset, particularly to test platform compatibility ${ }^{22,25-29}$. Detailed information on the datasets is available in Table S1. Information on consensus molecular subtype (CMS) classification was obtained from the Colorectal Cancer Subtyping Consortium for all array datasets ${ }^{2}$. For cases with missing CMS information, CMS labels were inferred by using the random forest (RF) method provided by the CMSclassifier R package ${ }^{2}$. Genes covered in both of the discovery and validation datasets were used for further analysis.

\section{Development of the MAP predictor}

Development of a gene-based predictor (MAPgene)

A schematic drawing of the MAP development process is provided in Fig. S1. To select informative genes for MSI prediction, we first identified differentially expressed genes (DEGs) between MSI and MSS tumors using the Wilcoxon rank-sum test in the discovery cohort. To 
construct and train a prediction model, RNA-seq data were divided into training and internal validation datasets at a ratio of 4 to 1 . To extract the most discriminative genes from the DEGs, the recursive feature elimination-random forest (RFE-RF) algorithm was used on the 466 training dataset. Briefly, feature selection was conducted by the backward selection method, wherein the RFE-RF repeatedly constructed an RF model by eliminating features with the least importance. The selection process was repeated 100 times, applying an upsampling approach to the MSI group (due to the small group size) using caret $^{30}$ and randomForest $\mathrm{R}$ package. The final model (MAPgene) was then selected based on that with the best area under a receiver operating characteristic curve (AUC) for 31 genes.

Development of an absolute, gene-pair-based predictor (MAPpairs)

To make the MAPgene model absolute (i.e., to predict MSI status from a single patient without comparison to a reference cohort or sample-wise normalization), a new model (MAPpairs) was 100 times. The final absolute model was selected according to its AUC.

Development of a tumor microenvironment-integrated model (MAPsig)

To construct a more sophisticated model, we exploited the molecular differences in cancer-, immune-, and TME-related signatures between MSI and MSS tumors. We collected 101 
signatures, including immune and stromal cells (TCIA and MCP-counter) ${ }^{31,32}$, cancer hallmarks from $\mathrm{MSigDB}^{33}$, immune-related signatures, such as epithelial and mesenchymal signatures $^{34}$; stromal and immune signatures $^{35}$; immunoinhibitory signatures and immunostimulatory signatures) ${ }^{31}$; T-cell-inflamed gene expression profile (GEP) ${ }^{36}$ and IFN- $\gamma$ expanded signatures ${ }^{36}$; cell cycle signature ${ }^{37}$; cell cycle regulator ${ }^{38}$; mismatch repair (KEGG), C-ECM signature ${ }^{39}$; angiogenesis, HLA class I and II family signature ${ }^{40}$; pro-inflammatory cytokines and chemokines ${ }^{40}$; CD8 T cells (Teff) ${ }^{41}$; and the MAP signature. To obtain signature scores for each individual sample, single sample gene-set enrichment analysis (ssGSEA), with ssgsea.norm $=\mathrm{F}$, was applied for the signatures above. Additionally, for cross-platform comparability, the acquired score was adjusted to a value between 1 to 10 . We used the same modeling method as that for the MAPgene and MAPpairs models, although with different input values. Finally, the MAPsig model and features were selected for inclusion in the final according to those that provided the best AUC.

\section{Model refinement}

When applying the MAPpairs model, we noted that true MSI samples tended to be classified with MSI at a probability much higher than $70 \%$. Thus, only samples with a probability of having MSI that was more than $70 \%$ were assigned MSI status. Samples with a predicted probability of MSI that was lower than $70 \%$ were further examined by applying the MAPsig model to determine final MSI status, as it showed high overall AUC, accuracy, and specificity, but low sensitivity, making it of use in only verifying a true MSS sample. The software is available at https://sourceforge.net/p/mapmsi/wiki/MAP/.

\section{Validation dataset}

To evaluate the predictive performance of the MAP model, we employed RNA-seq data for 
364 CRCs $(N=106)$ with $\log _{2}$-transformed rsem.norm data. Also, to assess platform independency and the applicability of MAP on different array datasets, we collected data for five cohorts. In the microarray datasets, the probes per gene were selected using Jetset (http://www.cbs.dtu.dk/biotools/jetset/) ${ }^{42}$. The array datasets were processed using fRMA R package per sample ${ }^{43}$. A total of five datasets were evaluated for the following: accuracy, sensitivity, specificity, F1 score, and balanced accuracy. All information on the datasets is provided in Table S1. For RNA-seq of stomach adenocarcinoma (STAD), and uterine corpus endometrial carcinoma (UCEC) were downloaded from the TCGA data portal (https://portal.gdc.cancer.gov/).

\section{Consistency of genes in a microsatellite instability classifier model based on gene} expression

To verify the consistency of feature genes with discriminative value in an MSI classifier model using gene expression, the Wilcoxon rank-sum test was used to analyze the external RNA-seq validation dataset. In addition, to assess the utility of MAP for MSI prediction, we calculated MAP signature scores (31-gene-set signature) using ssGSEA and compared them between MSI and MSS groups, as well as among MSI CMSs, using the Wilcoxon rank-sum test and KruskalWallis test.

\section{MSI signature construction at UCEC and STAD}

To investigate the MSI signature that can distinguish MSS and MSI in each cancer types, the same method was applied when constructing the MAP signature, except that the $P<0.02$ and $\mid \log _{2}$ fold change $\mid>1$ criteria was applied to identify sufficient number of DEG from two types of cancer. TCGA-UCEC and STAD expression dataset were download TCGA-STAD and 
390 https://gdc.cancer.gov/about-data/publications/panimmune. In this file, only 12 of 15

391 signatures of preMSIm existed. The missing genes were HENMT1, NOL4L, and RTF2.

392

\section{Statistical analysis}

394 Comparisons of two groups were conducted using the Wilcoxon rank-sum test, while 395 comparisons of multiple groups were performed using the Kruskal-Wallis test. All statistical 396 analyses were conducted using R language software (https://www.r-project.org/). 
This research was supported by a grant of the Korea Health Technology R\&D Project through the Korea Health Industry Development Institute (KHIDI), funded by the Ministry of Health \& Welfare, Republic of Korea (grant number HI14C1324).

403

404

Author Information

405

Affiliations

406

Department of Biomedical Systems Informatics and Brain Korea 21 PLUS Project for

Medical Science, Yonsei University College of Medicine, Seoul 03722, South Korea

408

Mi-Kyoung Seo, Hyundeok Kang, Sangwoo Kim

409

Department of Nuclear Medicine, Seoul National University Hospital, Seoul 03082, South

410

Korea

411

Mi-Kyoung Seo

412

413 Author Contributions

414 Conceptualization, M-K.S.; methodology, M-K.S.; software, M-K.S.; validation, M-K.S.; formal analysis, M-K.S.; investigation, M-K.S.; resources, M-K.S., and S.K.; data curation, M.S.; writing-original draft preparation, M-K.S., and H.K.; writing-review and editing, M-K.S., and H.K.; visualization, M-K.S.; supervision, S.K.; project administration, M-K.S.; funding acquisition, S.K. All authors have read and agreed to the published version of the manuscript.

Corresponding authors

421

Correspondence to Sangwoo Kim 
424 Ethics approval and consent to participate

425 Not applicable. No permissions were required to use any of the repository data. All methods 426 were performed in accordance with the PRISMA guidelines.

427

428 Conflicts of Interest

429 The authors declare no conflict of interest.

430

431 Data Availability

432 All relevant datasets used in the current study are available in the TCGA 433 (https://www.cancer.gov/about-nci/organization/ccg/research/structural-genomics/tcga) and 434 GEO (https://www.ncbi.nlm.nih.gov/geo/). This study analysis used all publicly available 435 datasets, and the dataset accession numbers included in Table S1. The software is available at $436 \mathrm{https}: / /$ sourceforge.net/p/mapmsi/wiki/MAP/. 


\section{References}

4381 Evrard, C., Tachon, G., Randrian, V., Karayan-Tapon, L. \& Tougeron, D. Microsatellite 439 Instability: Diagnosis, Heterogeneity, Discordance, and Clinical Impact in Colorectal $440 \quad$ Cancer. Cancers (Basel) 11, doi:10.3390/cancers11101567 (2019).

4412 Guinney, J. et al. The consensus molecular subtypes of colorectal cancer. Nat Med 21, $442 \quad$ 1350-1356, doi:10.1038/nm.3967 (2015).

4433 Cortes-Ciriano, I., Lee, S., Park, W. Y., Kim, T. M. \& Park, P. J. A molecular portrait of 444 microsatellite instability across multiple cancers. Nat Commun 8, 15180, 445 doi:10.1038/ncomms15180 (2017).

4464 Le, D. T. et al. PD-1 Blockade in Tumors with Mismatch-Repair Deficiency. N Engl J Med $447 \quad 372,2509-2520$, doi:10.1056/NEJMoa1500596 (2015).

4485 Sveen, A. et al. Colorectal Cancer Consensus Molecular Subtypes Translated to Preclinical 449 Models Uncover Potentially Targetable Cancer Cell Dependencies. Clin Cancer Res 24, 450 794-806, doi:10.1158/1078-0432.CCR-17-1234 (2018).

4516 Danaher, P. et al. Gene expression markers of Tumor Infiltrating Leukocytes. $J$ 452 Immunother Cancer 5, 18, doi:10.1186/s40425-017-0215-8 (2017).

4537 Kautto, E. A. et al. Performance evaluation for rapid detection of pan-cancer microsatellite 454 instability with MANTIS. Oncotarget 8, 7452-7463, doi:10.18632/oncotarget.13918 455 (2017).

4568 Bonneville, R. et al. Landscape of Microsatellite Instability Across 39 Cancer Types. JCO 457 Precis Oncol 2017, doi:10.1200/PO.17.00073 (2017).

4589 Niu, B. et al. MSIsensor: microsatellite instability detection using paired tumor-normal 459 sequence data. Bioinformatics 30, 1015-1016, doi:10.1093/bioinformatics/btt755 (2014).

46010 Hause, R. J., Pritchard, C. C., Shendure, J. \& Salipante, S. J. Classification and 
characterization of microsatellite instability across 18 cancer types. Nat Med 22, 13421350, doi:10.1038/nm.4191 (2016).

11 Li, L., Feng, Q. \& Wang, X. PreMSIm: An R package for predicting microsatellite instability from the expression profiling of a gene panel in cancer. Comput Struct Biotechnol J 18, 668-675, doi:10.1016/j.csbj.2020.03.007 (2020).

467

12 Danaher, P. et al. A gene expression assay for simultaneous measurement of microsatellite instability and anti-tumor immune activity. $J$ Immunother Cancer 7, 15, doi:10.1186/s40425-018-0472-1 (2019).

13 Pacinkova, A. \& Popovici, V. Cross-platform Data Analysis Reveals a Generic Gene

14 Giraldo, N. A. et al. The clinical role of the TME in solid cancer. Br J Cancer 120, 45-53, doi:10.1038/s41416-018-0327-z (2019). (2015).

18 Klingbiel, D. et al. Prognosis of stage II and III colon cancer treated with adjuvant 5-

15 Darst, B. F., Malecki, K. C. \& Engelman, C. D. Using recursive feature elimination in random forest to account for correlated variables in high dimensional data. BMC Genet 19, 65, doi:10.1186/s12863-018-0633-8 (2018).

16 Giordano, G. et al. JAK/Stat5-mediated subtype-specific lymphocyte antigen 6 complex, locus G6D (LY6G6D) expression drives mismatch repair proficient colorectal cancer. $J$ Exp Clin Cancer Res 38, 28, doi:10.1186/s13046-018-1019-5 (2019).

17 Hesson, L. B. et al. Lynch syndrome associated with two MLH1 promoter variants and allelic imbalance of MLH1 expression. Hum Mutat 36, 622-630, doi:10.1002/humu.22785 fluorouracil or FOLFIRI in relation to microsatellite status: results of the PETACC-3 trial. Ann Oncol 26, 126-132, doi:10.1093/annonc/mdu499 (2015). 
486

487

488

489

490

491

492

493

494

495

496

497

498

499

500

501

502

503

504

505

506

507

508

509

510

19 Seo, M. K., Paik, S. \& Kim, S. An Improved, Assay Platform Agnostic, Absolute Single Sample Breast Cancer Subtype Classifier. Cancers (Basel) 12, doi:10.3390/cancers12123506 (2020).

20 Auslander, N. et al. Robust prediction of response to immune checkpoint blockade therapy in metastatic melanoma. Nat Med 24, 1545-1549, doi:10.1038/s41591-018-0157-9 (2018).

21 Chang, S. C. et al. Clinicopathological and Molecular Profiles of Sporadic Microsatellite Unstable Colorectal Cancer with or without the CpG Island Methylator Phenotype (CIMP). Cancers (Basel) 12, doi:10.3390/cancers12113487 (2020).

22 Vasaikar, S. et al. Proteogenomic Analysis of Human Colon Cancer Reveals New Therapeutic Opportunities. Cell 177, 1035-1049 e1019, doi:10.1016/j.cell.2019.03.030 (2019).

23 Popat, S., Hubner, R. \& Houlston, R. S. Systematic review of microsatellite instability and colorectal cancer prognosis. J Clin Oncol 23, 609-618, doi:10.1200/JCO.2005.01.086 (2005).

24 Kim, C. G. et al. Effects of microsatellite instability on recurrence patterns and outcomes in colorectal cancers. Br J Cancer 115, 25-33, doi:10.1038/bjc.2016.161 (2016).

25 Marisa, L. et al. Gene expression classification of colon cancer into molecular subtypes: characterization, validation, and prognostic value. PLoS Med 10, e1001453, doi:10.1371/journal.pmed.1001453 (2013).

26 de Sousa, E. M. F. et al. Methylation of cancer-stem-cell-associated Wnt target genes predicts poor prognosis in colorectal cancer patients. Cell Stem Cell 9, 476-485, doi:10.1016/j.stem.2011.10.008 (2011).

27 Barras, D. et al. BRAF V600E Mutant Colorectal Cancer Subtypes Based on Gene Expression. Clin Cancer Res 23, 104-115, doi:10.1158/1078-0432.CCR-16-0140 (2017).

28 Jorissen, R. N. et al. DNA copy-number alterations underlie gene expression differences 
between microsatellite stable and unstable colorectal cancers. Clin Cancer Res 14, 80618069, doi:10.1158/1078-0432.CCR-08-1431 (2008).

29 Cancer Genome Atlas, N. Comprehensive molecular characterization of human colon and rectal cancer. Nature 487, 330-337, doi:10.1038/nature11252 (2012).

30 Kuhn, M. Building Predictive Models in R Using the caret Package. Journal of Statistical Software 28 (2008).

51731 Charoentong, P. et al. Pan-cancer Immunogenomic Analyses Reveal GenotypeCell Rep 18, 248-262, doi:10.1016/j.celrep.2016.12.019 (2017).

32 Becht, E. et al. Estimating the population abundance of tissue-infiltrating immune and stromal cell populations using gene expression. Genome Biol 17, 218, doi:10.1186/s13059-016-1070-5 (2016).

33 Liberzon, A. et al. The Molecular Signatures Database (MSigDB) hallmark gene set collection. Cell Syst 1, 417-425, doi:10.1016/j.cels.2015.12.004 (2015).

34 Linnekamp, J. F. et al. Consensus molecular subtypes of colorectal cancer are recapitulated in in vitro and in vivo models. Cell Death Differ 25, 616-633, doi:10.1038/s41418-0170011-5 (2018).

35 Yoshihara, K. et al. Inferring tumour purity and stromal and immune cell admixture from expression data. Nat Commun 4, 2612, doi:10.1038/ncomms3612 (2013).

36 Ayers, M. et al. IFN-gamma-related mRNA profile predicts clinical response to PD-1 blockade. J Clin Invest 127, 2930-2940, doi:10.1172/JCI91190 (2017).

37 Davoli, T., Uno, H., Wooten, E. C. \& Elledge, S. J. Tumor aneuploidy correlates with markers of immune evasion and with reduced response to immunotherapy. Science $\mathbf{3 5 5}$, doi:10.1126/science.aaf8399 (2017).

8 Cancer Genome Atlas Research, N. Comprehensive molecular characterization of 
urothelial bladder carcinoma. Nature 507, 315-322, doi:10.1038/nature12965 (2014).

537

538

539

540

541

542

543

544

545

546

547

548

549

550

551

552

39 Chakravarthy, A., Khan, L., Bensler, N. P., Bose, P. \& De Carvalho, D. D. TGF-betaassociated extracellular matrix genes link cancer-associated fibroblasts to immune evasion and immunotherapy failure. Nat Commun 9, 4692, doi:10.1038/s41467-018-06654-8 (2018).

40 Tamborero, D. et al. A Pan-cancer Landscape of Interactions between Solid Tumors and Infiltrating Immune Cell Populations. Clin Cancer Res 24, 3717-3728, doi:10.1158/10780432.CCR-17-3509 (2018).

41 Rosenberg, J. E. et al. Atezolizumab in patients with locally advanced and metastatic urothelial carcinoma who have progressed following treatment with platinum-based chemotherapy: a single-arm, multicentre, phase 2 trial. Lancet 387, 1909-1920, doi:10.1016/S0140-6736(16)00561-4 (2016).

42 Li, Q., Birkbak, N. J., Gyorffy, B., Szallasi, Z. \& Eklund, A. C. Jetset: selecting the optimal microarray probe set to represent a gene. BMC Bioinformatics 12, 474, doi:10.1186/14712105-12-474 (2011)

43 McCall, M. N., Bolstad, B. M. \& Irizarry, R. A. Frozen robust multiarray analysis (fRMA). Biostatistics 11, 242-253, doi:10.1093/biostatistics/kxp059 (2010). 


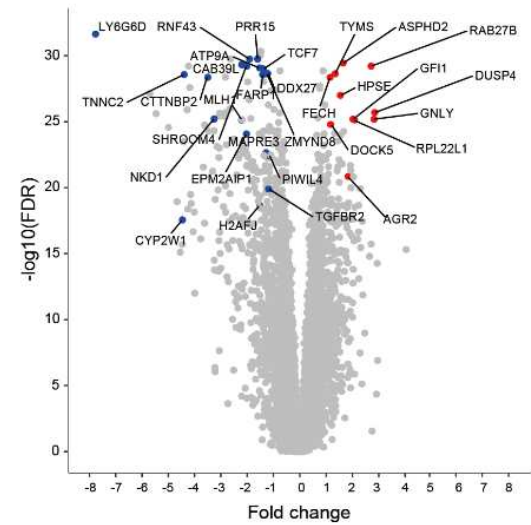

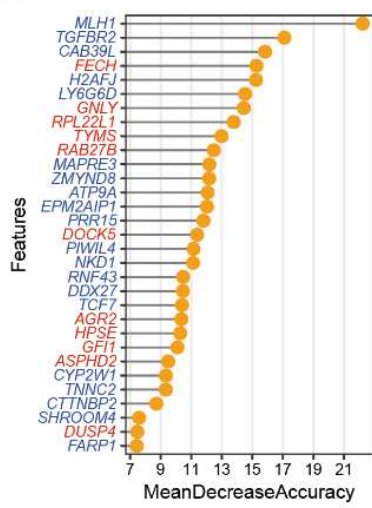

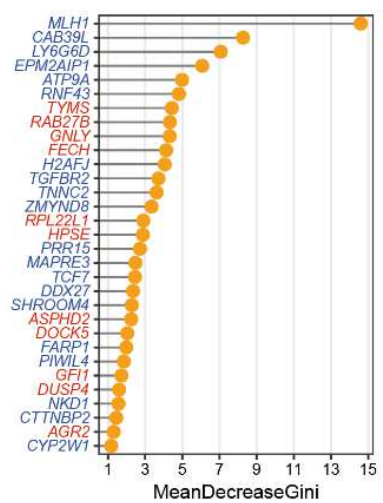

MeanDecreaseGini

C
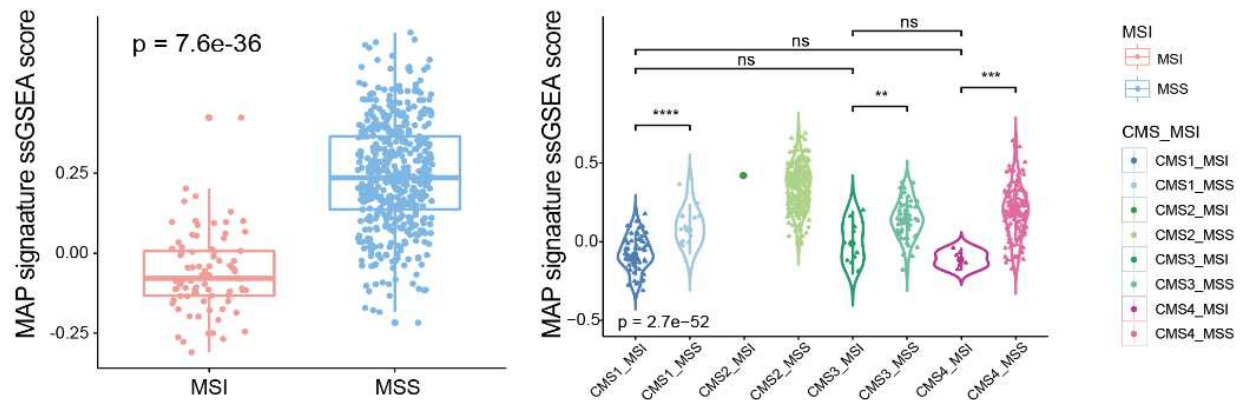

Figure 1. Designing the MAP signature from RFE-RF analysis of gene expression data. (a) A

volcano plot for DEGs between MSI and MSS samples. The $\mathrm{x}$ axis represents $\log _{2}$ fold changes in gene expression data for MSI versus MSS samples. Colored dots are significant DEGs in MAP signature; red and blue indicate up- and downregulated genes, respectively. (b) The importance of 31 features is based on accuracy and Gini index scores. The mean decrease in accuracy is a measure of how much influence it has in improving classification accuracy. The mean decrease in Gini is a measure of how impurity can be reduced by features used when separating nodes. The genes with red and blue colors indicate up- and downregulated genes in MSI, compared with MSS, respectively. (c) MAP signature. A box-plot of MAP signature ssGSEA scores according to MSI status (left) and CMS-MSI and MSS subtypes (right). The dots represent samples. MAP signature scores differ significantly between MSI and MSS samples independent of CMS subtypes. CMS2-MSI did not confirm statistical significance 
578 because the number of samples was small. $* P<0.05, * * P<0.01, * * * P<0.005$. DEG ;

579 differentially expressed gene, CMS ; consensus molecular subtype, ssGSEA ; single-sample 580 gene set enrichment analysis, FDR ; false discovery rate.

581

582 


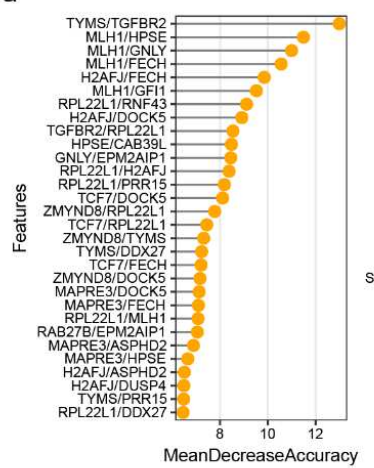

C
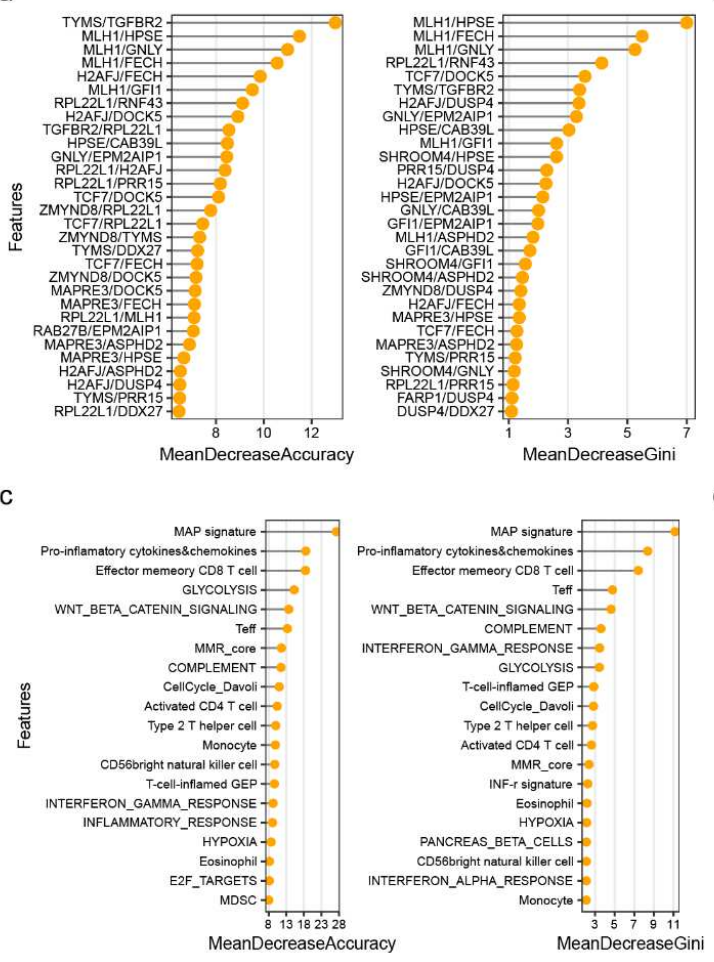
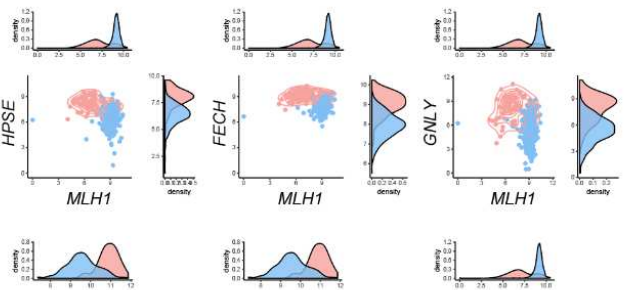

斯

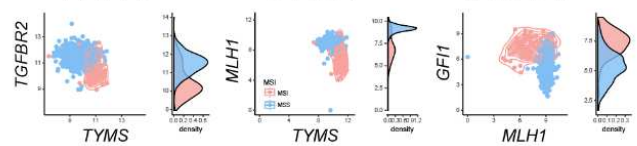

d
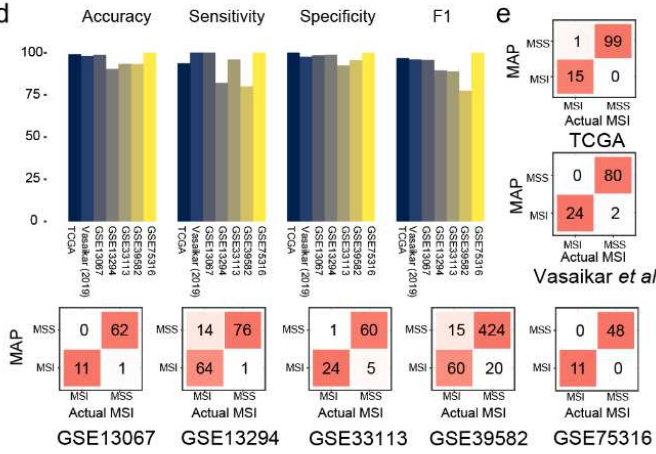

Figure 2. MAP model. (a) Top 30 important features of the MAPpairs model. The mean

585

586

587

588

589

590

591

592

593

594 decrease in accuracy (left) is a measure of how much influence a feature has in improving classification accuracy. The mean decrease in Gini (right) is a measure of how impurity can be reduced by features used when separating nodes. (b) A scatter plot and histogram of the gene pairs. MLH1-related rules and TFGBR2/TYMS rule are shown. (c) Top 20 important features (signatures) of the MAPsig model. (d) Performance (accuracy, sensitive, specificity, and F1) of the MAP model. (e) Confusion matrices of the validation dataset. The actual MSI means MSI status provided in the dataset study. The red color-scale reflects percentages of class predictions against the actual class. 

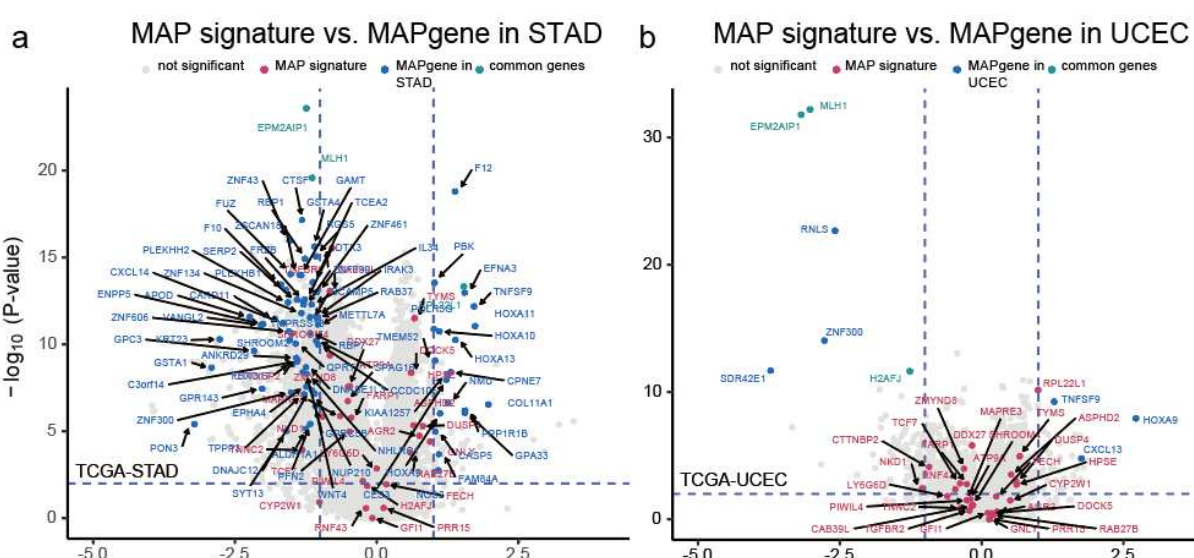

596

597

598

599

600

601

c
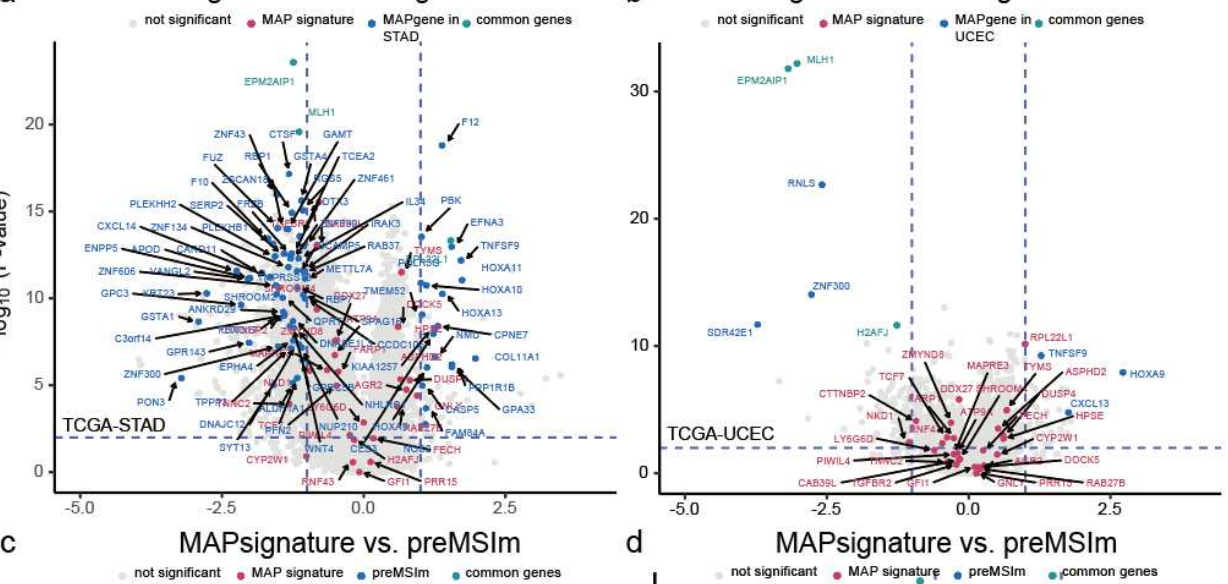

602

603

604

605

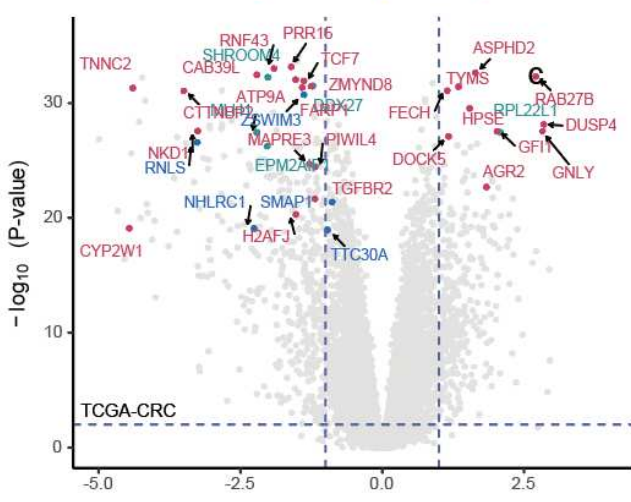

606

607

e MAPsignature vs. preMSIm

e MAPsignature vs. preMSIm

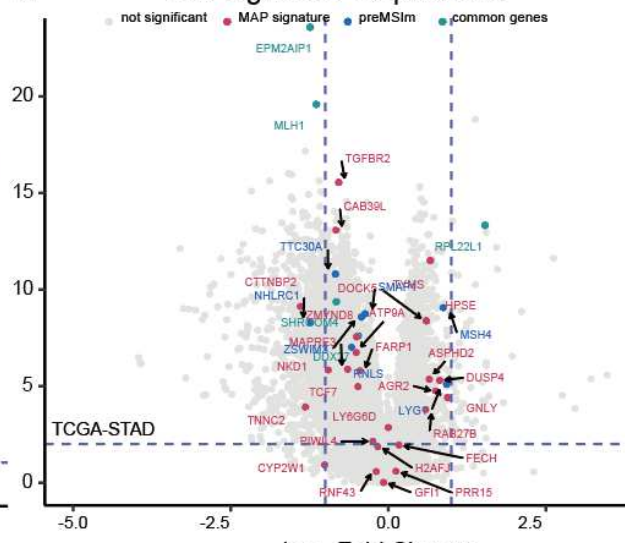

$\log _{2}$ Fold Change

608

609

610

611

612

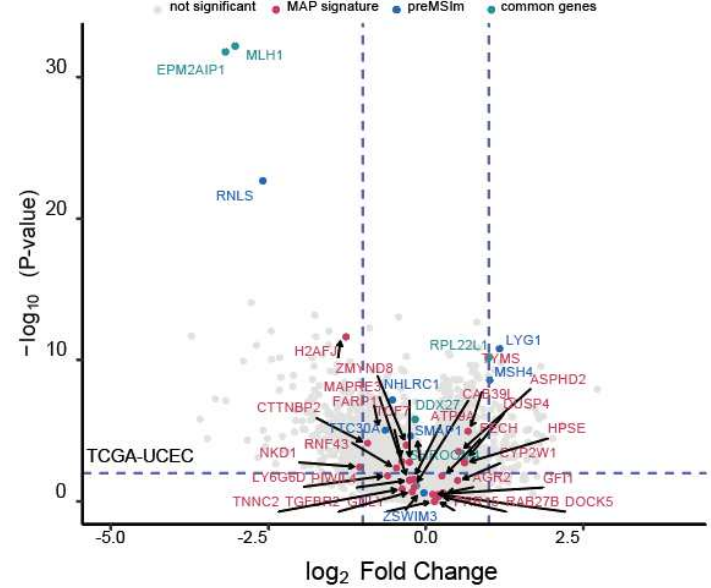

614 Figure 3. MSI signatures. (a) MAP signature and UCEC MSI signature on TCGA-UCEC. (b)

615 MAP signature and STAD MSI signature on TCGA-STAD (c) MAP signature and preMSIm

616 signature on TCGA-COADREAD. (d) MAP signature and preMSIm signature on TCGA-

617 STAD. (e) MAP signature and preMSIm signature on TCGA-UCEC. The x-axis represents

$618 \log _{2}$ fold changes in gene expression data for MSI versus MSS samples. The colored dots mean 
619 the genes of the corresponding signatures marked in each panel. The blue dotted line on the x620 axis means -1 and 1 of the $\log _{2}$ fold change scale, and $2\left(-\log _{10}(0.01)\right)$ on the $y$-axis. 


\section{Supplementary Files}

This is a list of supplementary files associated with this preprint. Click to download.

- MAPsupplementaryv1.pdf 